\title{
Multiwavelength study of the infrared dust bubble S51
}

\author{
C. P. Zhang ${ }^{1,2,3}$ and J. J. Wang ${ }^{1,2}$ \\ 1 National Astronomical Observatories, Chinese Academy of Sciences, 100012 Beijing, PR China \\ e-mail: zcp0507@gmail.com \\ 2 NAOC-TU Joint Center for Astrophysics, 850000 Lhasa, PR China \\ 3 Graduate University of the Chinese Academy of Sciences, 100080 Beijing, PR China
}

Received 5 January 2012 / Accepted 13 June 2012

\begin{abstract}
Aims. We investigate the environment of the infrared dust bubble S51 and search for evidence of triggered star formation in its surroundings.

Methods. We performed a multiwavelength study of the region around S51 with data taken from large-scale surveys: 2MASS, GLIMPSE, MIPSGAL, IRAS, and MALT90. We analyzed the spectral profile and the distribution of the molecular gas $\left({ }^{13} \mathrm{CO}, \mathrm{C}^{18} \mathrm{O}\right.$, $\mathrm{HCN}, \mathrm{HNC}, \mathrm{HCO}^{+}, \mathrm{C}_{2} \mathrm{H}, \mathrm{N}_{2} \mathrm{H}^{+}$, and $\mathrm{HC}_{3} \mathrm{~N}$ ), and dust in the environment of S51. We used a mid-infrared emission three-color image to explore the physical environment and GLIMPSE color-color diagram [5.8]-[8.0] versus [3.6]-[4.5] to search for young stellar objects and identify the ionizing star candidates.

Results. From a comparison of the morphology of the molecular gas and the Spitzer $8.0 \mu \mathrm{m}$ emission, we conclude that the dust bubble is interacting with $\mathrm{CO}$ at a kinematic distance of $3.4 \mathrm{kpc}$. The bubble $\mathrm{S} 51$ structure, carried with shell and front side, is exhibited with ${ }^{13} \mathrm{CO}$ and $\mathrm{C}^{18} \mathrm{O}$ emission. Both outflow and inflow may exist in sources in the shell of bubble S51. We discover a small bubble G332.646-0.606 $\left(R_{\text {in }}=26^{\prime \prime}, r_{\text {in }}=15^{\prime \prime}, R_{\text {out }}=35^{\prime \prime}\right.$ and $\left.r_{\text {out }}=25^{\prime \prime}\right)$ located at the northwest border of S51. A water maser, a methanol maser, and IRAS 16158-5055 are located at the junction of the two bubbles. Several young stellar objects are distributed along an arc-shaped structure near the S51 shell. They may represent a second generation of stars whose formation was triggered by the bubble expanding into the molecular gas.
\end{abstract}

Key words. infrared: stars - stars: formation - ISM: bubbles - HII regions

\section{Introduction}

Churchwell et al. (2006, 2007) detected and cataloged about 600 mid-infrared dust (MIR) bubbles between longitudes $-60^{\circ}$ and $+60^{\circ}$. The bubbles have bright $8.0 \mu \mathrm{m}$ shells that enclose bright $24 \mu \mathrm{m}$ interiors. The infrared (IR) dust bubbles may be produced by exciting O- and/or B-type stars, which are located inside the bubble. The ultraviolet (UV) radiation from exciting stars may heat dust and ionize the gas to form an expanding bubble shell (Watson et al. 2008), which is known as the "collectand-collapse" process. This process can trigger the massive star formation near the shell clumps. These bubbles present an important opportunity to study the interaction between HII regions and molecular clouds.

We selected the IR dust bubble S51 from the catalog of Churchwell et al. (2006). S51 is a complete (closed ring) IR dust bubble centered on $l=332.673, b=-0.618$. It lies in the southern part of RCW106 (Mookerjea et al. 2004; Wong et al. 2008; Lo et al. 2009). The radius of the shell is $1.48^{\prime}$ (1.47 pc), and the average thickness of the shell is $0.32^{\prime}(0.32 \mathrm{pc})$, while the eccentricity of the ellipse is 0.82 . The assumed distance is $3.4 \mathrm{kpc}$.

MALT90 is a pilot survey conducted with the Mopra telescope in preparation for the Millimeter Astronomy Legacy Team Survey at $90 \mathrm{GHz}$ (Foster et al. 2011). One of the aims of this multimolecular line mapping in this work is to examine how different molecules correlate with each other and with bubble $\mathrm{S} 51$. The molecules selected include ${ }^{13} \mathrm{CO}, \mathrm{C}^{18} \mathrm{O}, \mathrm{HCN}, \mathrm{HNC}$, $\mathrm{HCO}^{+}, \mathrm{C}_{2} \mathrm{H}, \mathrm{N}_{2} \mathrm{H}^{+}$, and $\mathrm{HC}_{3} \mathrm{~N} .{ }^{13} \mathrm{CO}$ has an optical depth of 1 to 12 (Wong et al. 2008), but $\mathrm{C}^{18} \mathrm{O}$ can provide optical depth and line profile information $\left(n_{\text {crit }}=3 \times 10^{3} \mathrm{~cm}^{-3}\right)$. MALT90 emission traces dense gas, and each molecular transition provides slightly different information. For example, $\mathrm{HCN}\left(n_{\text {crit }}=2 \times 10^{5} \mathrm{~cm}^{-3}\right)$ traces high column density and is optically thick; $\mathrm{HNC}\left(n_{\text {crit }}=\right.$ $\left.3 \times 10^{5} \mathrm{~cm}^{-3}\right)$ is prevalent in cold gas $\left(n_{\text {crit }}=2 \times 10^{5} \mathrm{~cm}^{-3}\right)$; $\mathrm{HCO}^{+}\left(n_{\text {crit }}=4 \times 10^{5} \mathrm{~cm}^{-3}\right)$ often shows infall signatures and outflow wings (Rawlings et al. 2004; Fuller et al. 2005); $\mathrm{N}_{2} \mathrm{H}^{+}$ $\left(n_{\text {crit }}=4 \times 10^{5} \mathrm{~cm}^{-3}\right)$ is more resistant to freeze-out onto grains than the carbon-bearing species (Bergin et al. 2001); $\mathrm{HC}_{3} \mathrm{~N}$ is a good tracer of hot core chemistry.

In this work, we report a multiwavelength study of the environment surrounding the IR dust bubble S51. We aim to explore its surrounding interstellar medium (ISM) and search for signatures of star formation. We describe the data used in Sect. 2; the results and discussion are presented in Sect. 3; Sect. 4 summarizes the results.

\section{Data}

We analyzed IR and millimeter wavelength data extracted from the following large-scale surveys: the Two Micron All Sky Survey (2MASS) ${ }^{1}$ (Skrutskie et al. 2006), GLIMPSE (Benjamin et al. 2003; Churchwell et al. 2009), MIPSGAL (Carey et al. 2009), IRAS (Neugebauer et al. 1984), and MALT90 (Foster et al. 2011).

\footnotetext{
${ }^{1}$ 2MASS is a joint project of the University of Massachusetts and the Infrared Processing and Analysis Center/California Institute of Technology, funded by the National Aeronautics and Space Administration and the National Science Foundation.
} 


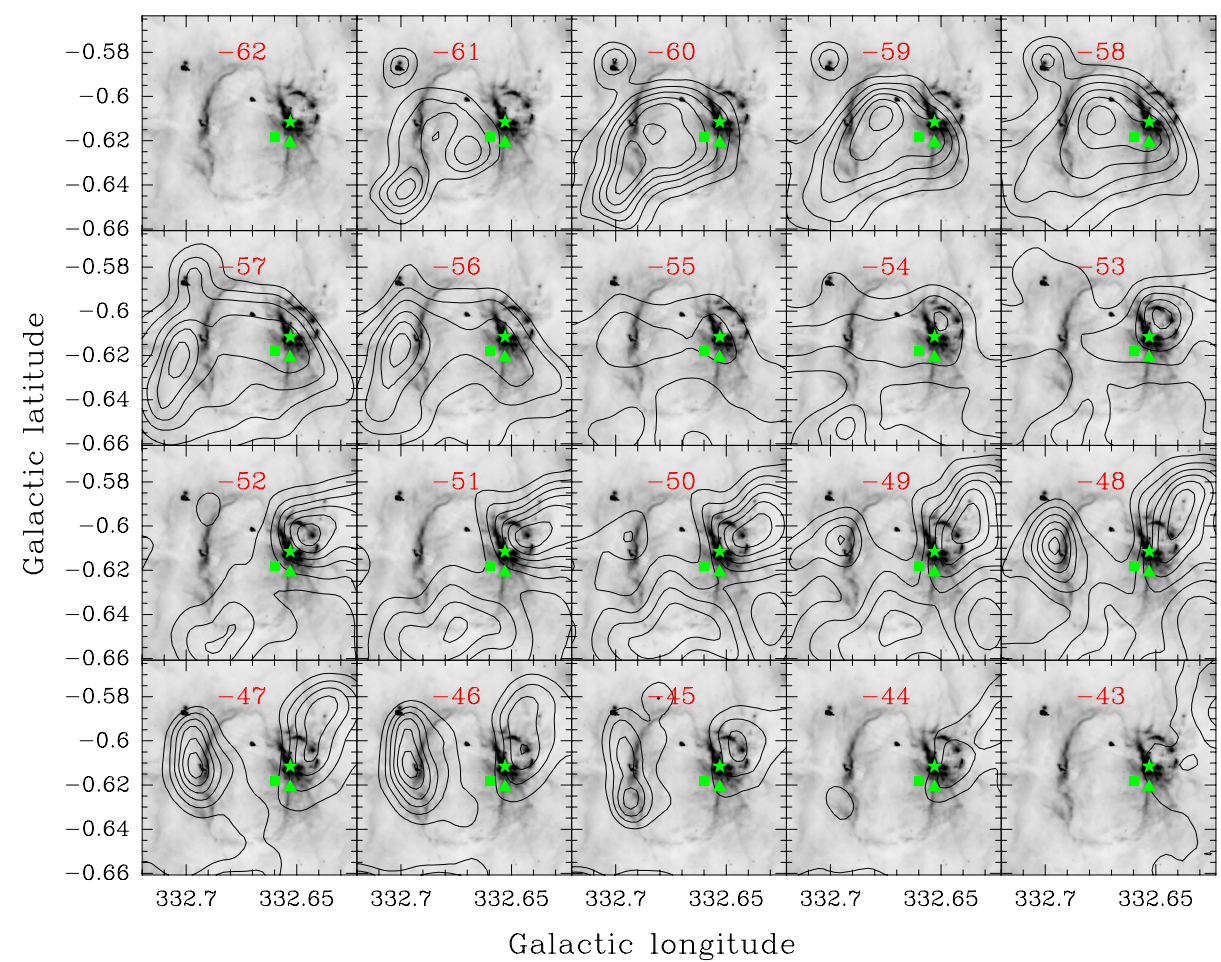

Fig. 1. Integrated velocity contours of the ${ }^{13} \mathrm{CO}$ emission every $1.0 \mathrm{~km} \mathrm{~s}^{-1}$ superimposed on the GLIMPSE $8.0 \mu \mathrm{m}$ grayscale. The contours are at multiples of the $13 \%$ level of each of the emission peaks. The peak of each mosaic from -62 to $-43 \mathrm{~km} \mathrm{~s}^{-1}$ is 0.718 , $1.632,3.584,7.503,9.191,11.388,14.901$, $16.428, \quad 10.915,9.673,12.091,15.416$, $16.916,16.467,14.378,12.772,6.043,3.018$, 1.535 , and $2.631 \mathrm{~K} \mathrm{~km} \mathrm{~s}^{-1}$, respectively. The green symbols " $\mathbf{\Delta}$ ", " $\mathbf{\square}$ " and " $\star$ " indicate the positions of the water maser, the methanol maser, and IRAS 16158-5055, respectively.
GLIMPSE is a MIR survey of the inner Galaxy performed with the Spitzer Space Telescope. We used the mosaicked images from GLIMPSE and the GLIMPSE Point-Source Catalog (GPSC) in the Spitzer-IRAC (3.6, 4.5, 5.8 and $8.0 \mu \mathrm{m})$. IRAC has an angular resolution between 1.5" and 1.9" (Fazio et al. 2004; Werner et al. 2004). MIPSGAL is a survey of the same region as GLIMPSE, using the MIPS instrument $(24$ and $70 \mu \mathrm{m})$ on Spitzer. The MIPSGAL resolution is $6^{\prime \prime}$ at $24 \mu \mathrm{m}$.

The Mopra 22-m radio telescope has a full width at half maximum (FWHM) beam size of $\sim 33^{\prime \prime}$ at $\sim 110 \mathrm{GHz}$ for ${ }^{13} \mathrm{CO} \mathrm{J}=$ $1-0$ and $\mathrm{C}^{18} \mathrm{O} J=1-0$ transitions. The correlator was configured with 1024 channels over a 64-MHz bandwidth, which provided a velocity resolution of $\sim 0.17 \mathrm{~km} \mathrm{~s}^{-1}$ per channel over a useable velocity bandwidth of $\sim 120 \mathrm{~km} \mathrm{~s}^{-1}$ (Lo et al. 2009; Bains et al. 2006; Wong et al. 2008). The observing was set up so that the central channel corresponded to $\sim 50 \mathrm{~km} \mathrm{~s}^{-1}$, at which the velocity of the emission from the GMC complex is centered.

The on-the-fly (OTF) mapping mode of Mopra was used for MALT90. Maps were made with the beam center running on a $3.4^{\prime} \times 3.4^{\prime}$ grid. The scan rate was $3.92^{\prime \prime}$ per second. The map was made with $12^{\prime \prime}$ spacing between adjacent rows, giving 17 rows per map. Because the Mopra beam at $90 \mathrm{GHz}$ is $38^{\prime \prime}$, this row spacing provides redundancy in the map. The full $8 \mathrm{GHz}$ bandwidth of the MOPra Spectrometer (MOPS) was split into 16 zoom bands of $138 \mathrm{MHz}$ each, providing a velocity resolution of $\sim 0.11 \mathrm{~km} \mathrm{~s}^{-1}$ in each band. In this work, six MALT90 pilot survey lines $\left(\mathrm{HCN}(J=1-0), \mathrm{HNC}(J=1-0), \mathrm{HCO}^{+}(J=1-0)\right.$, $\mathrm{C}_{2} \mathrm{H}(N=1-0, J=3 / 2-1 / 2, F=2-1), \mathrm{N}_{2} \mathrm{H}^{+}(J=1-0)$ and $\left.\mathrm{HC}_{3} \mathrm{~N}(J=10-9)\right)$ were used to trace the environment of bubble S51.

For the MOPRA observations, OFF positions were chosen at $\pm 1^{\circ}$ in Galactic latitude away from the plane (positive offset for sources at positive Galactic latitude and vice versa), and a single OFF position was observed for every two scan rows. Pointing on $\mathrm{SiO}$ masers was performed every $1-1.5 \mathrm{~h}$, maintaining pointing precision to better than about $10^{\prime \prime}$. The brightness temperature $T_{\mathrm{MB}}$ is related to the antenna temperature $T_{\mathrm{A}}^{*}$ by $T_{\mathrm{MB}}=T_{\mathrm{A}}^{*} / \eta_{v}$, where $\eta_{v}$ is the frequency-dependent beam efficiency. According to Ladd et al. (2005) and Lo et al. (2009), the main beam efficiency at $86 \mathrm{GHz}$ is $\eta_{86 \mathrm{GHz}}=0.49$, and at $110 \mathrm{GHz}$ is $\eta_{110 \mathrm{GHz}}=0.44$. The results presented in this paper are in terms of main bright temperature $T_{\mathrm{MB}}(\mathrm{K})$. The ${ }^{13} \mathrm{CO}$ and $\mathrm{C}^{18} \mathrm{O}$ data cubes were obtained from Lo et al. (2009). MALT90 data cubes were downloaded from the online archive ${ }^{2}$. The ${ }^{13} \mathrm{CO}, \mathrm{C}^{18} \mathrm{O}$ and MALT90 data cubes were processed with CLASS and GREG in the GILDAS software package ${ }^{3}$.

\section{Results and discussion}

The clue of this section is as follows. Firstly, by analyzing the channel maps (Figs. 1 and 2) of bubble S51 with ${ }^{13} \mathrm{CO}$ and $\mathrm{C}^{18} \mathrm{O}$ emission, we found that bubble $\mathrm{S} 51$ has shell and front side characters strongly associated with $8.0 \mu \mathrm{m}$ emission. The integration intensity maps of $[-62.0-43],.[-62.0-53.0]$ and $[-53.0-43.0]$ components are shown in Fig. 3. In addition, to examine the clumps of bubble S51, we used MALT90 multimolecular line mapping (Fig. A.1 in Appendix A) to compare them with each other and with IR emission distribution. The molecules include $\mathrm{HCN}, \mathrm{HNC}, \mathrm{HCO}^{+}, \mathrm{C}_{2} \mathrm{H}, \mathrm{N}_{2} \mathrm{H}^{+}$, and $\mathrm{HC}_{3} \mathrm{~N}$. And we also show the spectra of these molecules in Fig. 4. These spectra indicate that there are signs of inflow or outflow, whose velocity channels are shown in Fig. 5. Furthermore, we show two three-color images of MIR emission of IR dust bubble S51 with $3.6-4.5-8.0 \mu \mathrm{m}$ and $4.5-8.0-24 \mu \mathrm{m}$ in Fig. 6, so that we can understand the IR structure of bubble S51. Finally, we used GLIMPSE color-color diagram [5.8]-[8.0] versus [3.6]-[4.5] (Fig. 7) and spectral energy distribution (SED) fitting (Fig. 8) to search for young stars and identify exciting star candidates, whose distribution is shown in Fig. 9.

\footnotetext{
${ }^{2}$ http://atoa.atnf.csiro.au/MALT90/

3 http://iram.fr/IRAMFR/GILDAS/
} 


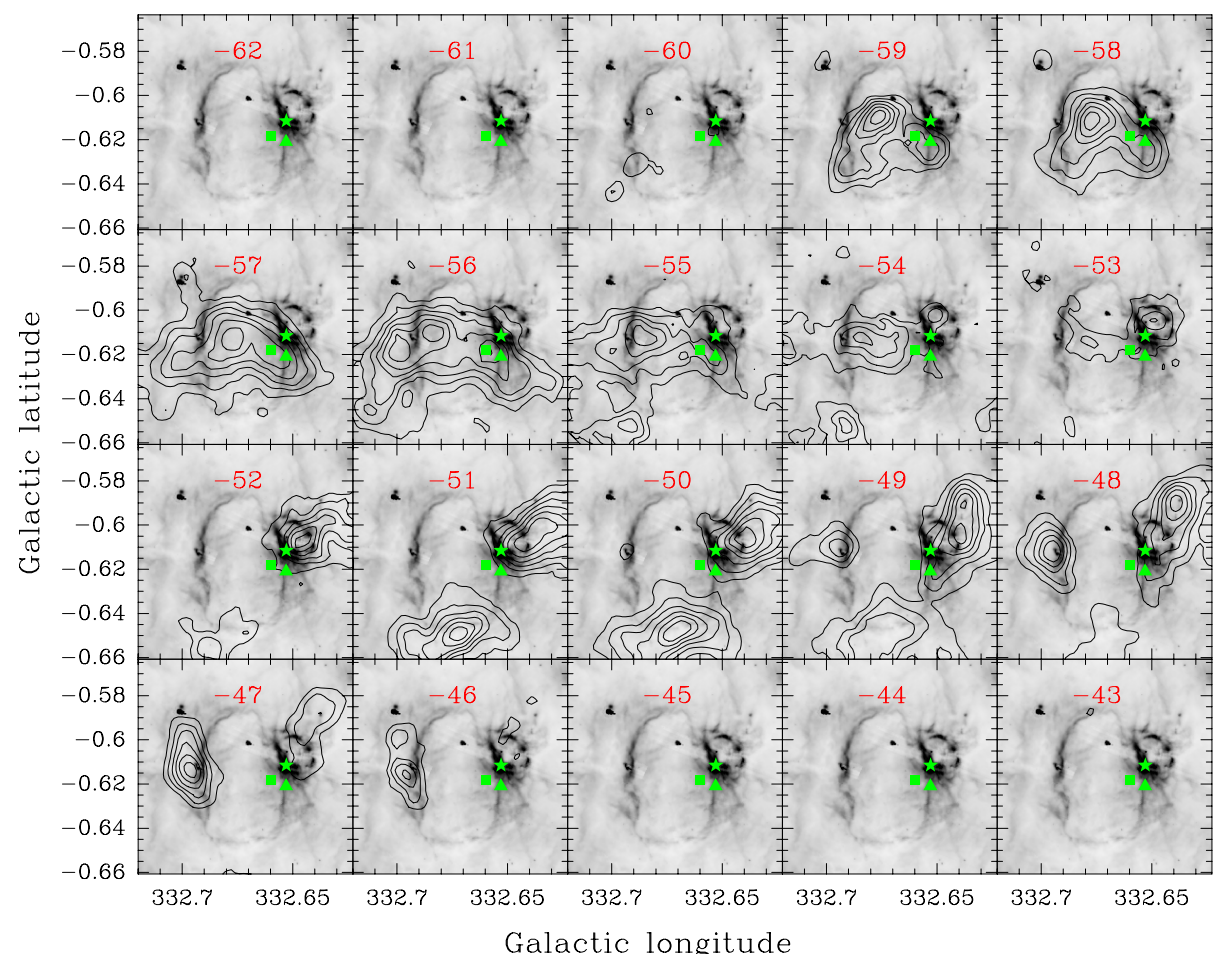

Galactic longitude

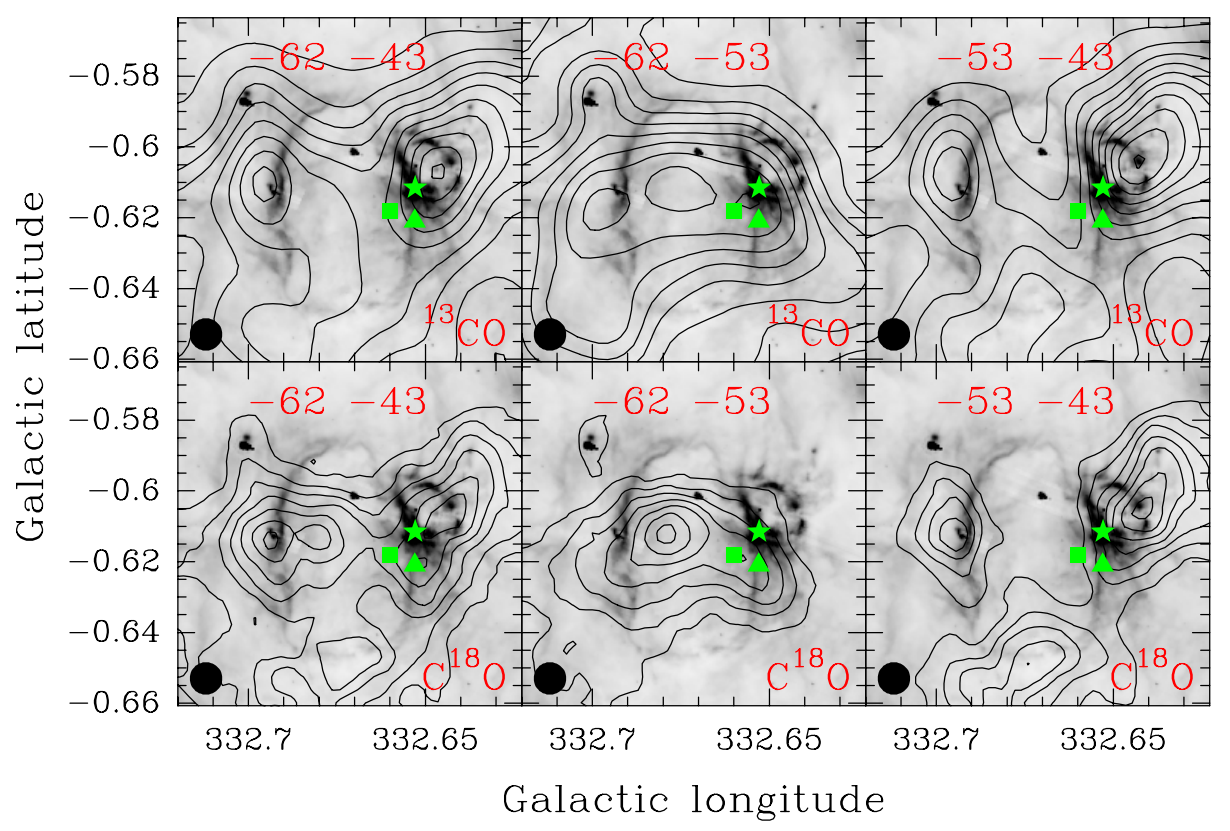

Fig. 2. Integrated velocity contours of the $\mathrm{C}^{18} \mathrm{O}$ emission every $1.0 \mathrm{~km} \mathrm{~s}^{-1}$ superimposed on the GLIMPSE $8.0 \mu \mathrm{m}$ grayscale. The contours are at multiples of the $13 \%$ level of each of the emission peaks. The peak of each mosaic from -62 to $-43 \mathrm{~km} \mathrm{~s}^{-1}$ is 0.518 , $0.590,0.870,2.625,4.342,4.081,3.663$, $2.814,1.627,1.529,2.671,3.697,4.914$, $4.572,4.514,3.756,1.524,0.575,0.558$, and $0.685 \mathrm{~K} \mathrm{~km} \mathrm{~s}^{-1}$, respectively. The green sym-

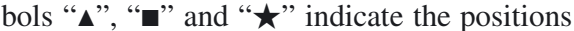
of the water maser, the methanol maser, and IRAS 16158-5055, respectively.

Fig. 3. Integrated velocity contours of the ${ }^{13} \mathrm{CO}$ (upper three mosaics) and $\mathrm{C}^{18} \mathrm{O}$ (lower three mosaics) emission superimposed on the GLIMPSE $8.0 \mu \mathrm{m}$ grayscale. The contours are at multiples of the $9 \%$ and $12 \%$ level of each of the ${ }^{13} \mathrm{CO}$ and $\mathrm{C}^{18} \mathrm{O}$ emission peaks, respectively. The peaks of upper three mosaics from left to right are 123.136, 59.055, and $93.380 \mathrm{~K} \mathrm{~km} \mathrm{~s}^{-1}$; the peaks of the lower three mosaics from left to right are 25.598, 18.456 , and $20.458 \mathrm{~K} \mathrm{~km} \mathrm{~s}^{-1}$, respectively. The integrated velocity range is indicated in

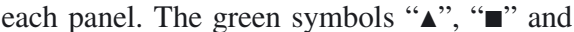
" $\star$ " indicate the positions of the water maser, the methanol maser, and IRAS 16158-5055, respectively.

\subsection{The shell and front side of bubble S51}

We inspected the molecular gas around S51 from the Mopra ${ }^{13} \mathrm{CO}$ and $\mathrm{C}^{18} \mathrm{O}$ emission in the whole velocity range and found two independent velocity components $([-62.0-53.0]$ and $[-53.0-43.0] \mathrm{km} \mathrm{s}^{-1}$ ) along the line of sight. Figures 1 and 2 show the integrated velocity maps of the ${ }^{13} \mathrm{CO}$ and $\mathrm{C}^{18} \mathrm{O}$ emission every $1.0 \mathrm{~km} \mathrm{~s}^{-1}$ between -62.5 and $-42.5 \mathrm{~km} \mathrm{~s}^{-1}$. The $8.0 \mu \mathrm{m}$ grayscale shows the position and size of S51.

Comparing the molecular and MIR morphologies, there are many interesting places among the $\left[\begin{array}{ll}-62.0 & -53.0\end{array}\right]$, $[-53.0-43.0] \mathrm{km} \mathrm{s}^{-1}$ components and $8.0 \mu \mathrm{m}$ emission, shown in Figs. 1-3. The morphology and peaks of $[-53.0-43.0] \mathrm{km} \mathrm{s}^{-1}$ component clearly correlate with $8.0 \mu \mathrm{m}$ emission. Accordingly, the $[-53.0-43.0] \mathrm{km} \mathrm{s}^{-1}$ component may be the shell of bubble S51. On the other hand, looking at the $-58.0 \mathrm{~km} \mathrm{~s}^{-1}$ panel in Fig. 2, we find that the contours fit quite well within the $8.0 \mu \mathrm{m}$ emission ring. The integration velocity map of the [-62.0 -53.0] km s${ }^{-1}$ component in Fig. 3 also shows that there is a good correlation between $\mathrm{C}^{18} \mathrm{O}$ contours and $8.0 \mu \mathrm{m}$ emission. We suggest that this cloud could be blueshifted gas associated with the front side of bubble S51. Since the $-48.0 \mathrm{~km} \mathrm{~s}^{-1}$ cloud is well associated with bubble shell, this bubble would be expanding at roughly $10.0 \mathrm{~km} \mathrm{~s}^{-1}$ along the line of sight. However, there is no redshifted counterpart.

From the ${ }^{13} \mathrm{CO}$ and $\mathrm{C}^{18} \mathrm{O}$ spectra of Fig. 4, we can also see that there are two velocity components at the locations of 

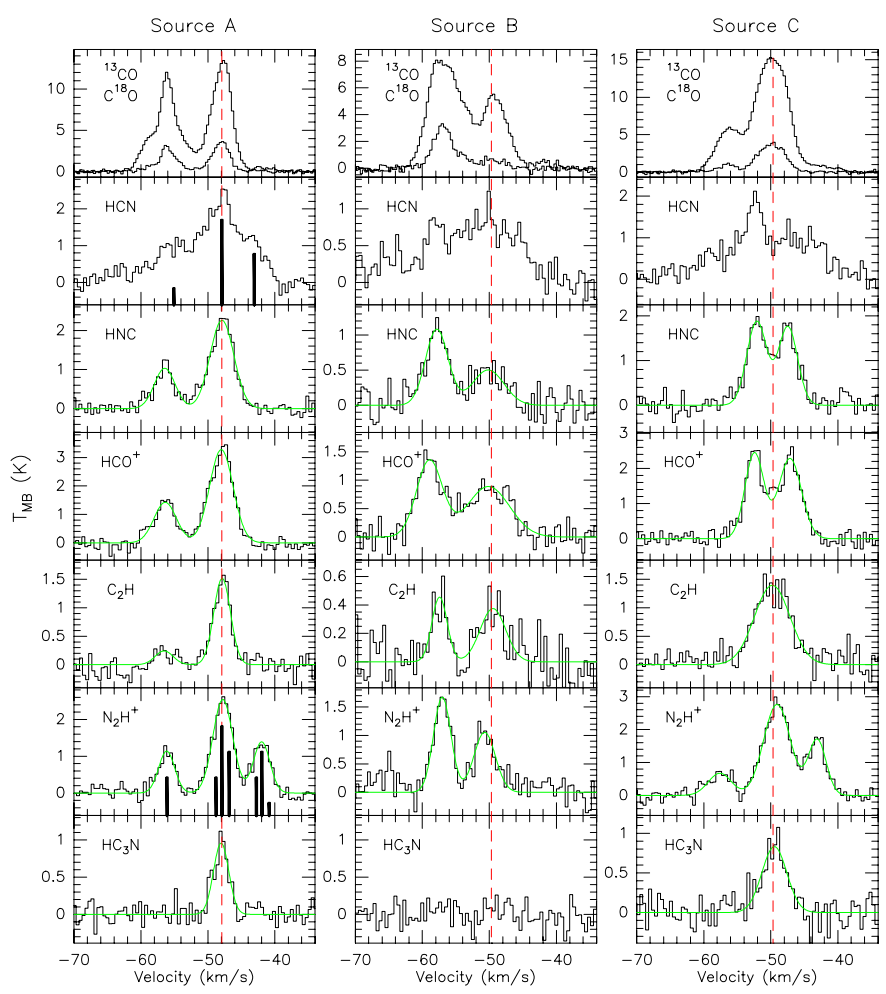

Fig. 4. MALT90 spectra of sources A, B and C, whose positions are represented in Fig. 9 with "A" $(l=332.693, b=-0.610)$, "B" $(l=$ $332.677, b=-0.619)$ and "C" $(l=332.645, b=-0.608)$, respectively. The red dashed lines indicate the peak velocities of the $\mathrm{C}^{18} \mathrm{O}$ emission. The green curves are Gauss-fit lines. $\mathrm{HCN}$ and $\mathrm{N}_{2} \mathrm{H}^{+}$have hyperfine splitting, shown as a black bar.

sources A, B and C (see Fig. 9), where source A is the core of eastern lobe of S51, source B lies at the front side or inside S51, and source $\mathrm{C}$ is the core of western lobe of S51. It is obvious that the spectral intensity of the $[-62.0-53.0] \mathrm{km} \mathrm{s}^{-1}$ component at the front side of bubble is stronger than that of $[-53.0-43.0] \mathrm{km} \mathrm{s}^{-1}$. At the shell of bubble S51, however, it is just the reverse. This case is also evidence that bubble S51 has a shell and front side structure.

For the $[-53.0-43.0] \mathrm{km} \mathrm{s}^{-1}$ component, the optical thin $\mathrm{C}^{18} \mathrm{O}$ emission contours and spectra (Figs. 2 and 4) show that the systematic velocity is $\sim-50.0 \mathrm{~km} \mathrm{~s}^{-1}$. Assuming the galactic rotation model of Fich et al. (1989) (with $R_{\odot}=8.5 \mathrm{kpc}$ and $\Theta_{\odot}=220 \mathrm{~km} \mathrm{~s}^{-1}$ ), we obtain kinematic distances of either 3.4 or $11.7 \mathrm{kpc}$. Lockman (1979) suggested that RCW106 lies the near kinematic distance after examining $\mathrm{H}_{2} \mathrm{CO}$ absorption spectra seen against the HII region continuum. Therefore, we adopted the near kinematic distance of $3.4 \mathrm{kpc}$ in this work.

\subsection{Opacity, excitation temperature, and column density}

We used the approach of Wong et al. (2008) to derive the opacity, excitation temperature, and column density of ${ }^{13} \mathrm{CO}$ and $\mathrm{C}^{18} \mathrm{O}$ within the box shown in Fig. A.1 of Appendix A. The size of the box is $4.3^{\prime} \times 4.3^{\prime}$ (where $4.3^{\prime}$ is the diameter of S51). The velocity range included is just $V=\sim-53.0$ to $\sim-43.0 \mathrm{~km} \mathrm{~s}^{-1}$ of bubble shell, and the box is centered on $l=332.673, b=-0.618$.

The relation between opacities and the ratio of ${ }^{13} \mathrm{CO}$ to $\mathrm{C}^{18} \mathrm{O}$ main-beam brightness temperature (Myers et al. 1983) is

$\frac{T_{\mathrm{MB}}\left({ }^{13} \mathrm{CO}\right)}{T_{\mathrm{MB}}\left(\mathrm{C}^{18} \mathrm{O}\right)}=\frac{1-\exp \left(-\tau_{13}\right)}{1-\exp \left(-\tau_{18}\right)}=\frac{1-\exp \left(-7.4 \tau_{18}\right)}{1-\exp \left(-\tau_{18}\right)}$.
Equation (1) assumes $\tau_{13}=7.4 \tau_{18}$ (Wilson \& Rood 1994; Wong et al. 2008). Furthermore, the excitation temperature $T_{\mathrm{ex}}$ is derived from the equation of radiative transfer:

$$
\left\{\begin{array}{l}
T_{\mathrm{MB}}=f\left[J\left(T_{\mathrm{ex}}\right)-J\left(T_{\mathrm{bg}}\right)\right][1-\exp (-\tau)] \\
J(T)=T_{0} /\left[\exp \left(T_{0} / T\right)-1\right]
\end{array}\right.
$$

where $f$ is the beam filling factor, $T_{b g}=2.7 \mathrm{~K}$ and $T_{0}=h v / k=$ $5.29 \mathrm{~K}$ for the $1-0$ transition of ${ }^{13} \mathrm{CO}$ (Wong et al. 2008). And then, assuming ${ }^{13} \mathrm{CO}$ is optical thin, we obtain the molecular ${ }^{13} \mathrm{CO}$ column density $N\left({ }^{13} \mathrm{CO}\right)$ from the relation (Bourke et al. 1997)

$$
N\left({ }^{13} \mathrm{CO}\right)_{\mathrm{thin}}=\frac{T_{\mathrm{ex}}+0.88}{1-\exp \left(-5.29 / T_{\mathrm{ex}}\right)} \cdot \frac{2.42 \times 10^{14}}{J\left(T_{\mathrm{ex}}\right)-J\left(T_{\mathrm{bg}}\right)} \int T_{\mathrm{MB}}\left({ }^{13} \mathrm{CO}\right) \mathrm{d} v .
$$

Finally, assuming $\left[\mathrm{H}_{2} /{ }^{13} \mathrm{CO}\right]$ abundance ratio is $7 \times 10^{5}$ (Frerking et al. 1982), the molecular hydrogen column $N\left(\mathrm{H}_{2}\right)$ was calculated. The molecular cloud mass was estimated at $M_{\text {shell }} \sim$ $1.3 \times 10^{4} M_{\odot}$ for the bubble shell at a distance of $D_{\mathrm{S} 51} \sim 3.4 \mathrm{kpc}$.

\subsection{Clump analysis}

To examine the clumps of bubble S51, we used multimolecular line mappings to compare them with each other and with the IR emission distribution.

In the box shown in Fig. A.1, the maximum and average column densities $N\left({ }^{13} \mathrm{CO}\right)$ are $9.72 \times 10^{17}$ and $5.98 \times$ $10^{16} \mathrm{~cm}^{-2}$, respectively. This figure also shows the main beam brightness temperature $T_{\mathrm{MB}}$, the brightness temperature ratio $T_{\mathrm{MB}}\left({ }^{13} \mathrm{CO}\right) / T_{\mathrm{MB}}\left(\mathrm{C}^{18} \mathrm{O}\right)$, the optical depth $\tau\left({ }^{13} \mathrm{CO}\right)$, and the excitation temperature $T_{\mathrm{ex}}\left({ }^{13} \mathrm{CO}\right)$. The distribution of these parameters (shown in color) are compared with contours showing the integrated intensity of different molecular transitions. The average $T_{\mathrm{MB}}\left({ }^{13} \mathrm{CO}\right) / T_{\mathrm{MB}}\left(\mathrm{C}^{18} \mathrm{O}\right), \tau\left({ }^{13} \mathrm{CO}\right)$ and $T_{\mathrm{ex}}\left({ }^{13} \mathrm{CO}\right)$ are 4.49 , 1.76 and 20.91, respectively. The peak positions of ${ }^{13} \mathrm{CO}, \mathrm{C}^{18} \mathrm{O}$ and $8.0 \mu \mathrm{m}$ emission are spatially coincident, and are situated at the east and west edge of the shell. The molecular clouds are extended around the $8.0 \mu \mathrm{m}$ emission peaks. At the south border of bubble S51, we can also see the shell of the filament structure from $8.0 \mu \mathrm{m}$ emission in Fig. 6. The ${ }^{13} \mathrm{CO}$ and $\mathrm{C}^{18} \mathrm{O}$ emission contours are distributed along the shell of the filament structure in Fig. 3. We also see from Fig. A.1 that the distribution of $T_{\mathrm{MB}}\left({ }^{13} \mathrm{CO}\right) / T_{\mathrm{MB}}\left(\mathrm{C}^{18} \mathrm{O}\right), \tau\left({ }^{18} \mathrm{CO}\right), T_{\mathrm{ex}}\left({ }^{13} \mathrm{CO}\right)$ and $\mathrm{N}\left({ }^{13} \mathrm{CO}\right)$ shows an arc-like morphology around the north edge of S51. However, it should be noted that these derived properties are not independent of each other.

$\mathrm{HCN}, \mathrm{HNC}, \mathrm{HCO}^{+}, \mathrm{C}_{2} \mathrm{H}$, and $\mathrm{N}_{2} \mathrm{H}^{+}$trace the position of highest density, while $\mathrm{HC}_{3} \mathrm{~N}$ traces the location of the hot core (Foster et al. 2011). We chose three sources (A, B and C) to analyze in Fig. 9; their spectra are shown in Fig. 4. Figure A.1 shows that a potentially dense and hot core is mainly located in the eastern and western shells. The spectra shown in Fig. 4 for the $[-53.0-43.0] \mathrm{km} \mathrm{s}^{-1}$ component demonstrate that source B inside S51 has a lower brightness temperature than sources A and $\mathrm{C}$ in the $\mathrm{S} 51$ shell. At source B, however, the MALT90 spectra of the $[-53.0-43.0] \mathrm{km} \mathrm{s}^{-1}$ component show the blueshifted velocity $[-62.0-53.0] \mathrm{km} \mathrm{s}^{-1}$, which is evidence that this is the front side of bubble S51. This suggests that the extended cloud of the $[-53.0-43.0] \mathrm{km} \mathrm{s}^{-1}$ component is scarce inside bubble S51, and the front side of bubble S51 has a rich abundance. However, the back side of bubble S51 is not shown in the MALT90 spectra. They also show that bubble S51 might be expanding at $10 \mathrm{~km} \mathrm{~s}^{-1}$ 
C. P. Zhang and J. J. Wang: Bubble S51
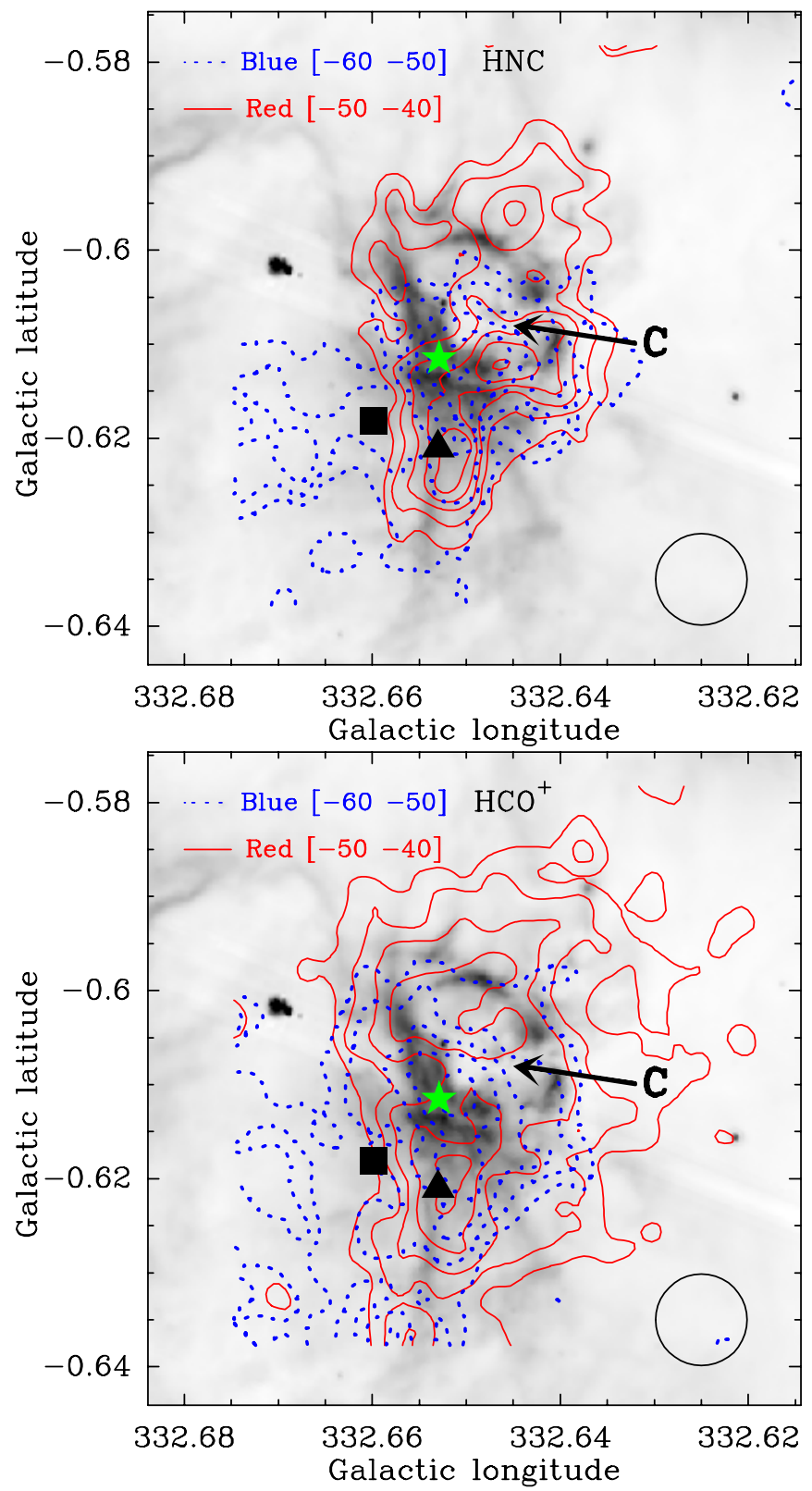

Fig. 5. Integrated velocity contours of $\mathrm{HNC}$ (top panel) and $\mathrm{HCO}^{+}$(bottom panel) emission superimposed on the GLIMPSE $8.0 \mu \mathrm{m}$ grayscale. The contours are at multiples of $11 \%, 11 \%, 12 \%$, and $12 \%$ level of the blue lobe of $\mathrm{HNC}$, the red lobe of $\mathrm{HNC}$, the blue lobe of $\mathrm{HCO}^{+}$, and the red lobe of $\mathrm{HCO}^{+}$emission peaks, which are 9.539, 8.618, 12.861, and $10.800 \mathrm{~K} \mathrm{~km} \mathrm{~s}^{-1}$, respectively. Source $\mathrm{C}$ is indicated with "C" in the image. The symbols " $\boldsymbol{\wedge}$ ", " $\mathbf{\square}$ " and " $\star$ " indicate the positions of the water maser, the methanol maser, and IRAS 16158-5055, respectively.

compared to the bubble shell along the line of sight. In addition, we note that IRAS $16158-5055$ is closer to the peaks of $\mathrm{N}_{2} \mathrm{H}^{+}$ and $\mathrm{HC}_{3} \mathrm{~N}$ than the water maser and the methanol maser.

\subsection{Inflow or outflow character}

As shown in Fig. 5, there is much trace of active star formation located in the shell of S51. The western shell of S51 contains a UCHII region, a water maser $(l=332.653, b=$ -0.621), a methanol maser $(l=332.660, b=-0.618)$, and IRAS 16158-5055 ( $l=332.653, b=-0.612)$ (Walsh et al. 1997; Breen et al. 2007). The water maser spectrum has three
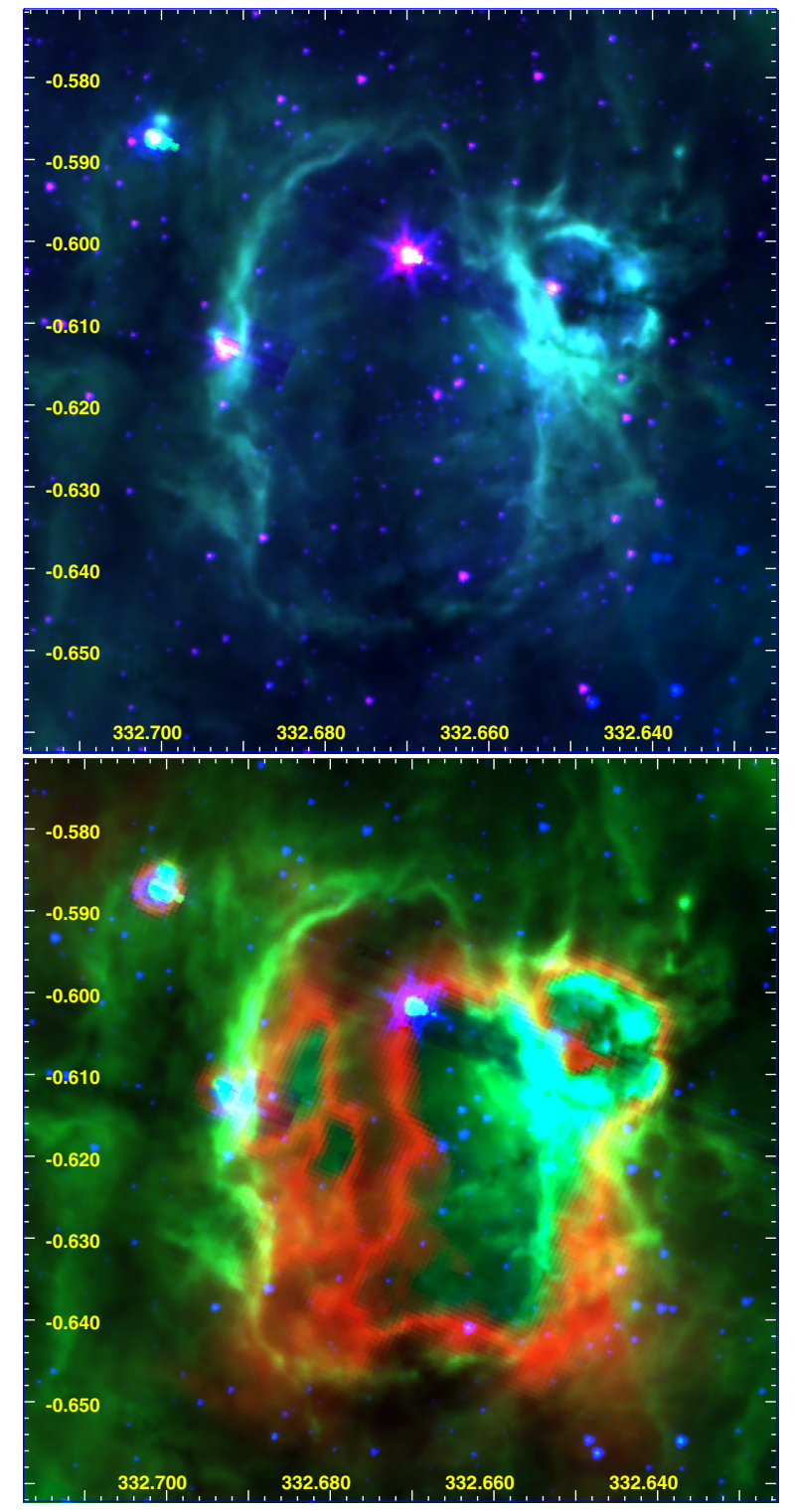

Fig. 6. MIR emission of IR dust bubble S51. Top: Spitzer-IRAC threecolor image $(3.6 \mu \mathrm{m}=$ red, $4.5 \mu \mathrm{m}=$ blue and $8.0 \mu \mathrm{m}=$ green $)$; bottom: Spitzer-IRAC and Spitzer-MIPSGAL three-color image $(4.5 \mu \mathrm{m}=$ blue, $8.0 \mu \mathrm{m}=$ green and $24 \mu \mathrm{m}=$ red). Note that the $24 \mu \mathrm{m}$ emission is saturated at the center of image.

peaks, with fluxes of $\sim 1 \mathrm{Jy}$ for the $-58.5 \mathrm{~km} \mathrm{~s}^{-1}$ component, $\sim 6 \mathrm{Jy}$ for $-47.5 \mathrm{~km} \mathrm{~s}^{-1}$, and $\sim 30 \mathrm{Jy}$ for $-45.2 \mathrm{~km} \mathrm{~s}^{-1}$, respectively (Walsh et al. 1997). The methanol maser spectrum has a velocity range of $-52 \sim-50 \mathrm{~km} \mathrm{~s}^{-1}$, and the peak flux is 7.1 Jy (Breen et al. 2007). This means that the $[-53.0-43.0] \mathrm{km} \mathrm{s}^{-1}$ component of the S51 and not the $[-62.0-53.0] \mathrm{km} \mathrm{s}^{-1}$ component of background is strongly correlated with the methanol and water masers.

On the west side of S51, the position of source $\mathrm{C}$ is marked in Fig. 5. Figure 4 shows the ${ }^{13} \mathrm{CO}, \mathrm{C}^{18} \mathrm{O}$ and MALT90 spectra of source $\mathrm{C}$. We marked the splittings of the hyperfine structure of $\mathrm{HCN}$ and $\mathrm{N}_{2} \mathrm{H}^{+}$with several black bars. The $[-62.0-53.0] \mathrm{km} \mathrm{s}^{-1}$ component is very faint in ${ }^{13} \mathrm{CO}, \mathrm{C}^{18} \mathrm{O}$ emission, as well as $\mathrm{HCN}, \mathrm{HNC}, \mathrm{HCO}^{+}, \mathrm{C}_{2} \mathrm{H}$, and $\mathrm{HC}_{3} \mathrm{~N}$. Consequently the $[-62.0-53.0] \mathrm{km} \mathrm{s}^{-1}$ component seems unrelated to the $[-53.0-43.0] \mathrm{km} \mathrm{s}^{-1}$ component in this cluster. Figure 4 also shows that the optically thick spectra of $\mathrm{HCN}$, 


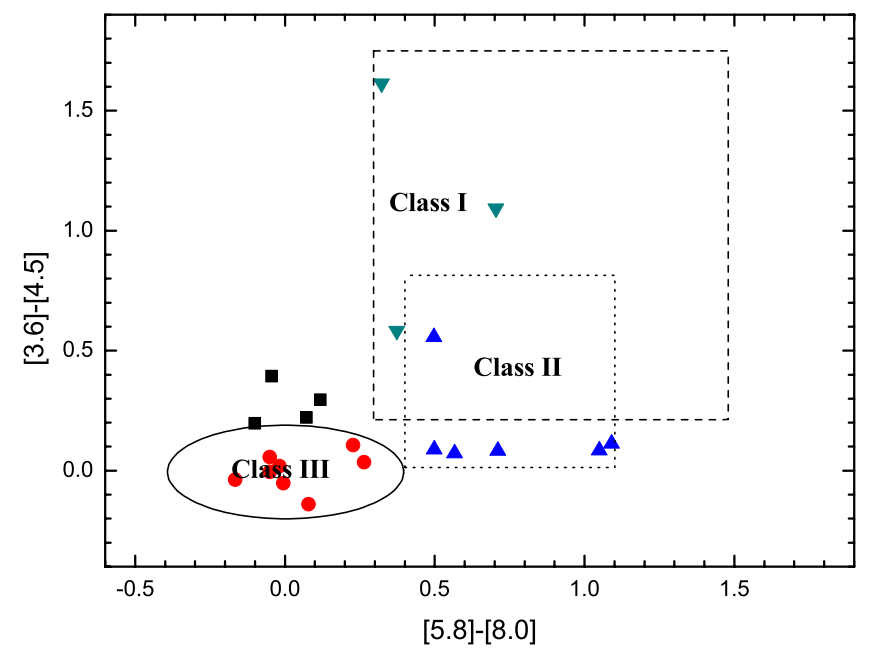

Fig. 7. GLIMPSE color-color diagram [5.8]-[8.0] versus [3.6]-[4.5]

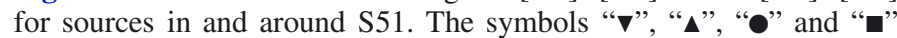
indicate the different stars of class I, II, III and others, respectively. The classification of class I, II and III indicates the stellar evolutionary stage as defined by Allen et al. (2004).

$\mathrm{HNC}$ and $\mathrm{HCO}^{+}$lines have absorption dips at the peak velocity $\left(-49.5 \mathrm{~km} \mathrm{~s}^{-1}\right)$ traced by $\mathrm{C}^{18} \mathrm{O}, \mathrm{C}_{2} \mathrm{H}, \mathrm{N}_{2} \mathrm{H}^{+}$and $\mathrm{HC}_{3} \mathrm{~N}$. The blue profile is stronger than the red profile shown from the $\mathrm{HCN}$ spectrum. Such a profile can be produced by inflow with cold gas wings and a hot central core (Mardones et al. 1997). Moreover, $\mathrm{HNC}$ and $\mathrm{HCO}^{+}$have broad line wings corresponding to $\mathrm{N}_{2} \mathrm{H}^{+}$ and $\mathrm{HC}_{3} \mathrm{~N}$, therefore source $\mathrm{C}$ could also be produced by outflow. Figure 5 shows the emission in two different velocity channels. The velocities of the water maser and the methanol maser are similar to the more negative velocity gas. We speculate that since this region is located at the junction of bubble S51 and bubble G332.646-0.606 (referred to Sect. 3.5), it is plausible that the interactions between the two bubbles or between the HII region and the molecular cloud have triggered this phenomenon.

\subsection{IR structure of $S 51$}

Figure 6 shows two Spitzer three-color images of S51. Both figures clearly show the PDR visible in $8.0 \mu \mathrm{m}$ (in green) emission, which originates mainly in the PAHs. Since these large molecules are destroyed inside the ionized region, the PAH emission delineates the HII region boundaries, and the molecular clouds are excited in the PDR by the radiation leaking from the HII region (Petriella et al. 2010; Pomarès et al. 2009). The $24 \mu \mathrm{m}$ emission (bottom panel in Fig. 6) appears to be inside bubble $\mathrm{S} 51$, and corresponds to hot dust. It is likely that $\mathrm{O}$ - and/or early B-type stars produced the bubble shell of this HII region, with hot dust located inside the bubble. The $3.6 \mu \mathrm{m}$ emission (in red, top panel in Fig. 6) shows the positions of the brightest stars.

We also note a small bubble G332.646-0.606, which is centered on $l=332.646, b=-0.606$ and located at the northwest edge of S51. The semimajor $\left(R_{\text {in }}\right)$ and semiminor $\left(r_{\text {in }}\right)$ axes of the inner ellipse are 26 and 15 arcsec, respectively; the semimajor $\left(R_{\text {out }}\right)$ and semiminor $\left(r_{\text {out }}\right)$ axes of the outer ellipse are 35 and 25 arcsec, respectively. From $8.0 \mu \mathrm{m}$ emission of bubble G332.646-0.606, the elliptical morphologic PDR shows a sketch of the bubble. Note that the $24 \mu \mathrm{m}$ emission is saturated inside the small bubble G332.646-0.606. At the junction between bubble S51 and G332.646-0.606, the IR emission is brighter than the rest of the shell; this is also true for the ${ }^{13} \mathrm{CO}, \mathrm{C}^{18} \mathrm{O}$ and MALT90

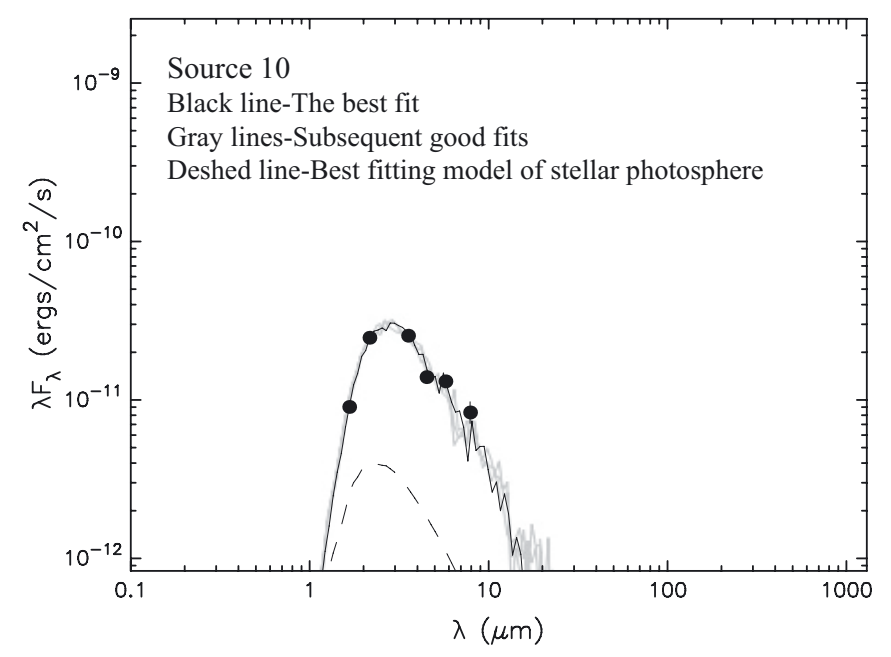

Fig. 8. SED fits of exciting star source $10(l=332.6664, b=-0.6266)$. The flux density of fitted data are the 2Mass $H$-band 5.01(0.22), the $K$-band 17.81(0.44), IRAC $3.6 \mu \mathrm{m} 30.16(1.26), 4.5 \mu \mathrm{m} \mathrm{20.93(1.48),}$ $5.8 \mu \mathrm{m} \mathrm{25.12(1.53),} \mathrm{and} 8.0 \mu \mathrm{m} \mathrm{22.15(3.46)} \mathrm{mJy}$, respectively.

emission lines. IRAS $16158-5055$ is also located at this position. It seems likely that this junction region is the birth space of several YSOs. In addition, the molecular gas of the bubble shell exhibits several clumps along the PDR (Fig. 6). The distribution and morphology of this material suggests that a collect-andcollapse process may be occurring.

\subsection{Search for young stars and identifying exciting stars}

The distribution of the IR point sources in the surroundings of S51 provides some signs of star formation. Figure 7 shows the [5.8]-[8.0] versus [3.6]-[4.5] color-color diagram for the sources extracted from the GLIMPSE Point Source Catalog in the Spitzer-IRAC bands in and around S51. We only considered sources with detection in the four Spitzer-IRAC bands. In Fig. 7, class I stars are protostars with circumstellar envelopes; class II are disk-dominated objects; and class III are main sequence and giant stars. The classification indicates the stellar evolutionary stage as defined by Allen et al. (2004).

Class I and class II stars are chosen to be the YSO candidates. The YSO candidates are drawn with symbols " $X$ " and " $\curlywedge$ " in Fig. 9, among which sources 4, 7, 8, 9, 13 and 17 near the shell of bubble are made up of an arc-shaped distribution. This may be caused by interaction between the HII region and molecular clouds, and be the production of a collect-and-collapse process.

We also tried to search for and identify exciting stars of S51. However, only sources 10 and 14 are located within the bubble. Source 14 belongs to a class I star, therefore we did not consider it to be an exciting star. Source 10 is a class II star, therefore we identified it in more detail with an SED fitting, using the tool developed by Robitaille et al. (2007), which is available online ${ }^{4}$. The good fitting models were selected according to the condition $\chi^{2}-\chi_{\min }^{2}<3$, where $\chi_{\min }^{2}$ is the minimum value of $\chi^{2}$ among all models.

In Fig. 8, we show the results for the SED fitting of our source 10 to the fluxes obtained from the GLIMPSE Point Source Catalog (Hora et al. 2008) and the 2MASS All-Sky Point Source Catalog (Skrutskie et al. 2006). We fit these sources allowing the extinction to range from 0 to $30 \mathrm{mag}$ and the distance

${ }^{4}$ http://caravan.astro.wisc.edu/protostars/ 


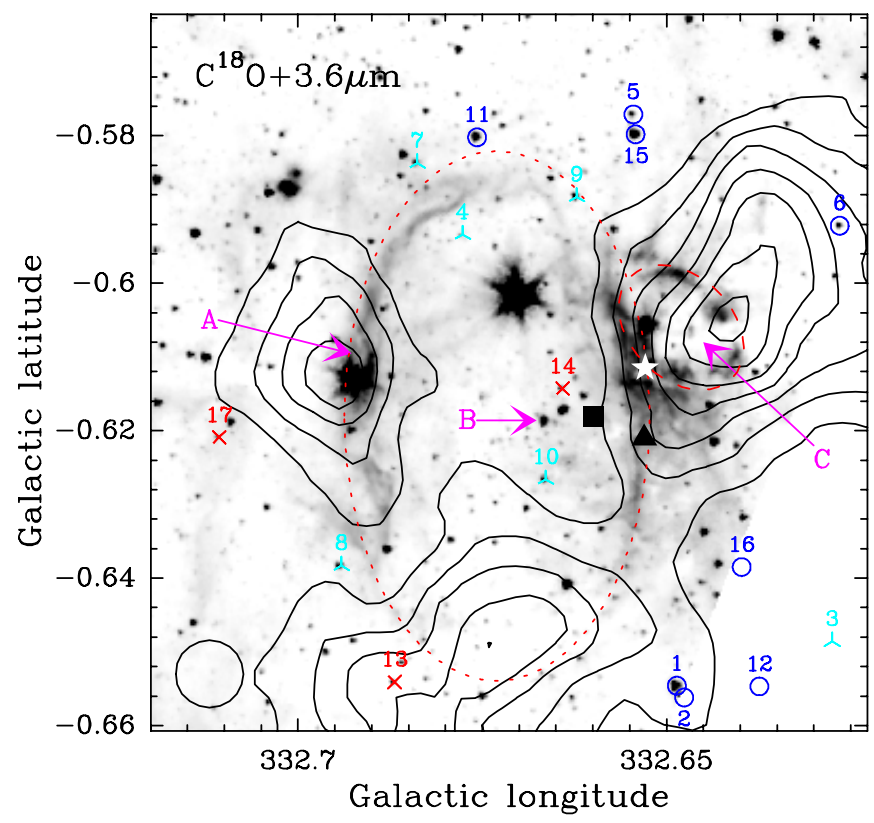

Fig. 9. Integrated velocity contours of the ${ }^{18} \mathrm{CO}$ emission superimposed on the GLIMPSE $3.6 \mu \mathrm{m}$ grayscale. The contours are at multiples of $12 \%$ level of the $\mathrm{C}^{18} \mathrm{O}$ emission peaks $93.380 \mathrm{~K} \mathrm{~km} \mathrm{~s}^{-1}$, and the integrated velocity range is from -53.0 to $-43.0 \mathrm{~km} \mathrm{~s}^{-1}$. Two dashed ellipses show the PAH morphology of the two bubbles. Letters "A" $(l=332.693, b=-0.610), " \mathrm{~B} "(l=332.677, b=-0.619)$ and "C" $(l=332.645, b=-0.608)$ indicate the chosen positions of three sources. The class I, II and III stars are indicated with symbols " $\times$ ", “人” and " $\bigcirc$ ". The symbols " $\mathbf{\Delta " , ~ " \square " ~ a n d ~ " ~} \star$ " indicate the positions of the water maser, the methanol maser, and IRAS point source, respectively.

to range from 3.0 to $4.0 \mathrm{kpc}$. The SED output shows that source 10 in class II is $\dot{M}_{\text {env }} / M_{\star}<10^{-6} \mathrm{yr}^{-1}$ and $M_{\text {disk }} / M_{\star}<10^{-6}$; the temperature of the center star is $31000 \mathrm{~K}$, in agreement with the effective temperature expected for an O-type star (Schaerer $\&$ de Koter 1997). Therefore, source 10 may be a candidate of the ionization of S51. Stellar winds emit from exciting stars. In addition, the presence of a central cavity in the distribution of the ionized gas may be a signature of the activities of stellar winds. The location of source 10 inside this cavity provides additional support for the assumption that this star is the most likely candidate to have created S51. Spectroscopic observation is needed to confirm this.

\section{Summary}

We have investigated the environment of the IR dust bubble S51 with several spectra $\left({ }^{13} \mathrm{CO}, \mathrm{C}^{18} \mathrm{O}, \mathrm{HCN}, \mathrm{HNC}, \mathrm{HCO}^{+}, \mathrm{C}_{2} \mathrm{H}\right.$, $\mathrm{N}_{2} \mathrm{H}^{+}$, and $\mathrm{HC}_{3} \mathrm{~N}$ ) and IR emission. The main results can be summarized as follows.

(1) We have distinguished two independent velocity components associated with S51 along the line of sight. One component belongs to the shell of bubble S51, and another may be the front side of bubble S51.

(2) We suggest that source $\mathrm{C}(l=332.645, b=-0.608)$ shows evidence of either outflow or inflow, located on the northwest border of S51.

(3) Next to the west border of bubble S51, we found a small bubble G332.646-0.606, whose dimensions are $R_{\text {in }}=26^{\prime \prime}$, $r_{\text {in }}=15^{\prime \prime}, R_{\text {out }}=35^{\prime \prime}$ and $r_{\text {out }}=25^{\prime \prime}$.

(4) MALT90 emission contours appear to be correlated with the S51 shell. The water maser, the methanol maser, and IRAS 16158-5055 are located in the shell. The YSO distribution is also correlated with ${ }^{13} \mathrm{CO}, \mathrm{C}^{18} \mathrm{O}$ and $8.0 \mu \mathrm{m}$ emission.

Acknowledgements. We wish to thank the anonymous referee for comments and suggestions, which improved the clarity of the paper. Particularly, we thank Nadia Lo for providing ${ }^{13} \mathrm{CO}$ and $\mathrm{C}^{18} \mathrm{O}$ data. This work was supported by the Young Researcher Grant of National Astronomical Observatories, Chinese Academy of Sciences No. O835032002.

\section{References}

Allen, L. E., Calvet, N., D’Alessio, P., et al. 2004, ApJS, 154, 363 Bains, I., Wong, T., Cunningham, M., et al. 2006, MNRAS, 367, 1609 Benjamin, R. A., Churchwell, E., Babler, B. L., et al. 2003, PASP, 115, 953 Bergin, E. A., Ciardi, D. R., Lada, C. J., Alves, J., \& Lada, E. A. 2001, ApJ, 557, 209

Bourke, T. L., Garay, G., Lehtinen, K. K., et al. 1997, ApJ, 476, 781

Breen, S. L., Ellingsen, S. P., Johnston-Hollitt, M., et al. 2007, MNRAS, 377, 491

Carey, S. J., Noriega-Crespo, A., Mizuno, D. R., et al. 2009, PASP, 121, 76

Churchwell, E., Povich, M. S., Allen, D., et al. 2006, ApJ, 649, 759

Churchwell, E., Watson, D. F., Povich, M. S., et al. 2007, ApJ, 670, 428

Churchwell, E., Babler, B. L., Meade, M. R., et al. 2009, PASP, 121, 213

Fazio, G. G., Hora, J. L., Allen, L. E., et al. 2004, ApJS, 154, 10

Fich, M., Blitz, L., \& Stark, A. A. 1989, ApJ, 342, 272

Foster, J. B., Jackson, J. M., Barnes, P. J., et al. 2011, ApJS, 197, 25

Frerking, M. A., Langer, W. D., \& Wilson, R. W. 1982, ApJ, 262, 590

Fuller, G. A., Williams, S. J., \& Sridharan, T. K. 2005, A\&A, 442, 949

Hora, J. L., Carey, S., Surace, J., et al. 2008, PASP, 120, 1233

Ladd, N., Purcell, C., Wong, T., \& Robertson, S. 2005, PASA, 22, 62

Lo, N., Cunningham, M. R., Jones, P. A., et al. 2009, MNRAS, 395, 1021

Lockman, F. J. 1979, ApJ, 232, 761

Mardones, D., Myers, P. C., Tafalla, M., et al. 1997, ApJ, 489, 719

Mookerjea, B., Kramer, C., Nielbock, M., \& Nyman, L.-Å. 2004, A\&A, 426, 119

Myers, P. C., Linke, R. A., \& Benson, P. J. 1983, ApJ, 264, 517

Neugebauer, G., Habing, H. J., van Duinen, R., et al. 1984, ApJ, 278, L1

Petriella, A., Paron, S., \& Giacani, E. 2010, A\&A, 513, A44

Pomarès, M., Zavagno, A., Deharveng, L., et al. 2009, A\&A, 494, 987

Rawlings, J. M. C., Redman, M. P., Keto, E., \& Williams, D. A. 2004, MNRAS, 351,1054

Robitaille, T. P., Whitney, B. A., Indebetouw, R., \& Wood, K. 2007, ApJS, 169, 328

Schaerer, D., \& de Koter, A. 1997, A\&A, 322, 598

Skrutskie, M. F., Cutri, R. M., Stiening, R., et al. 2006, AJ, 131, 1163

Walsh, A. J., Hyland, A. R., Robinson, G., \& Burton, M. G. 1997, MNRAS, 291, 261

Watson, C., Povich, M. S., Churchwell, E. B., et al. 2008, ApJ, 681, 1341

Werner, M. W., Roellig, T. L., Low, F. J., et al. 2004, ApJS, 154, 1

Wilson, T. L., \& Rood, R. 1994, ARA\&A, 32, 191

Wong, T., Ladd, E. F., Brisbin, D., et al. 2008, MNRAS, 386, 1069 


\section{Appendix A: Molecular line maps of bubble S51}

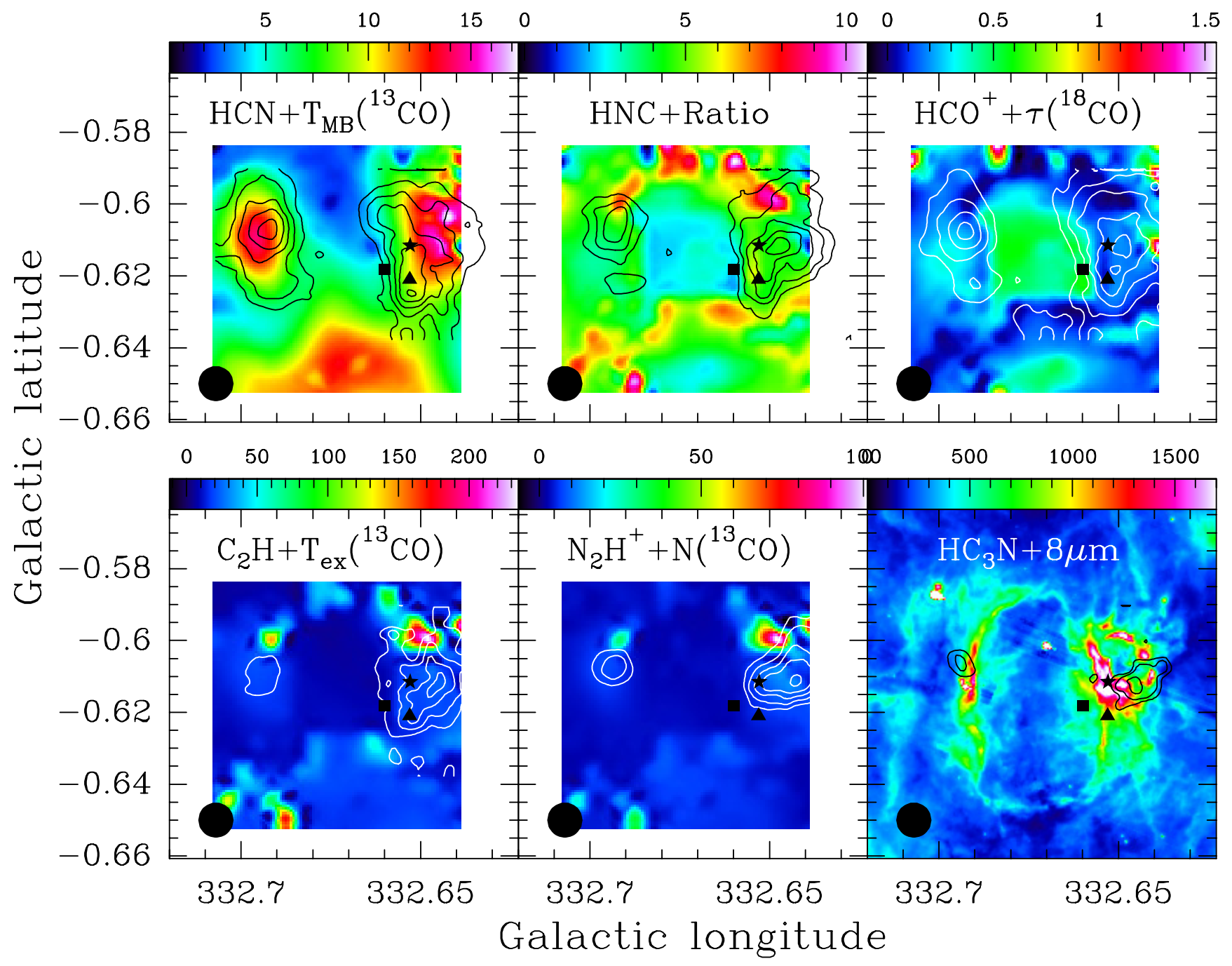

Fig. A.1. Integrated velocity contours of MALT90 emissions ( $\mathrm{HCN}, \mathrm{HNC}, \mathrm{HCO}^{+}, \mathrm{C}_{2} \mathrm{H}, \mathrm{N}_{2} \mathrm{H}^{+}$, and $\mathrm{HC}_{3} \mathrm{~N}$ ) superimposed on six color images of different parameter distributions $\left(T_{\mathrm{MB}}\left({ }^{13} \mathrm{CO}\right)\right.$, ratio $=T_{\mathrm{MB}}\left({ }^{13} \mathrm{CO}\right) / T_{\mathrm{MB}}\left(\mathrm{C}^{18} \mathrm{O}\right), \tau\left({ }^{18} \mathrm{CO}\right), T_{\mathrm{ex}}\left({ }^{13} \mathrm{CO}\right), N\left({ }^{13} \mathrm{CO}\right)\left[\times 10^{16}\right]$ and $\left.8 \mu \mathrm{m}\right)$. The contours are at multiples of the $12 \%, 13 \%, 12 \%, 16 \%, 14 \%$, and $15 \%$ level of each of the $\mathrm{HCN}, \mathrm{HNC}, \mathrm{HCO}^{+}, \mathrm{C}_{2} \mathrm{H}, \mathrm{N}_{2} \mathrm{H}^{+}$, and $\mathrm{HC}_{3} \mathrm{~N}$ emission peaks, which are $9.573,7.953,9.781,4.791,10.490$, and $2.555 \mathrm{~K} \mathrm{~km} \mathrm{~s}^{-1}$, respectively. The integrated velocity range is from $\sim-53.0$ to $\sim-43.0 \mathrm{~km} \mathrm{~s}{ }^{-1}$ for all

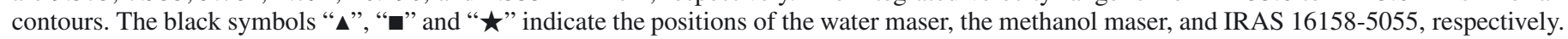

